\title{
Detecting Structural Changes in Whole Brain Based on Nonlinear Deformations-Application to Schizophrenia Research
}

\author{
Christian Gaser,* Hans-Peter Volz,* Stefan Kiebel „† Stefan Riehemann,* and Heinrich Sauer* \\ *Department of Psychiatry and †Department of Neurology, University of J ena, Philosophenweg 3, D-07740 J ena, Germany
}

Received J anuary 21, 1999

This paper describes a new method for detecting structural brain differences based on the analysis of deformation fields. Deformations are obtained by an intensity-based nonlinear registration routine that transforms one brain onto another one. We present a general multivariate statistical approach to analyze deformation fields in different subjects. This method was applied to the brains of 85 schizophrenic patients and 75 healthy volunteers to examine whether low frequency deformations are sufficiently sensitive to detect regional deviations in the brains of both groups. We observed significant changes caused by volume reduction in brains of schizophrenics bilaterally in the thalamus and in the superior temporal gyrus. On the left side, the superior frontal gyrus and precentral gyrus are found to be changed, while on the right side, the middle frontal gyrus was altered. In addition, there were significant changes in the occipital lobe (left lingual gyrus) and in the left cerebellum. Volume enlargement in brains of schizophrenics was observed in the right putamen and in the adjacent white matter of the thalamic region. Our data suggest a disturbance in the nodes of a prefrontal-thalamic-cerebellar circuitry. This provides further support for the model of "cognitive dysmetria," which postulates a disruption in these nodes. We have demonstrated the application of deformation-based morphometry by detecting structural changes in the whole brain. This technique is fully automatic, thus allowing for the inclusion of large samples, with no user bias or a priori-defined regions of interest. 1999 Academic Press

Key Words: MRI; brain; morphometry; nonlinear image registration; statistical parametric map; schizophrenia.

\section{INTRODUCTION}

Magnetic resonance imaging (MRI) studies have demonstrated considerable evidence that schizophrenia is associated with alterations in brain structure. Changes in cerebral structure affect diverse brain regions for example, the frontal, temporal, and limbic areas. However, abnormalities have not been consistently demonstrated (Lawrie and Abukmeil, 1998). There are many potential reasons for these inconsistent findings. The multiple and diverse symptoms of schizophrenia, issues such as sample size, medication effects, and the heterogeneity of the patients may be attributed to a number of problems. However, the lack of a proper definition of examined regions has most impact on the inconsistencies. Furthermore, in the majority of studies regions of interest (ROI) are segmented in consecutive slices either by hand or semiautomatically. This interactive approach introduces not only user bias, but is also highly time-consuming. This leads to the analysis of a limited number of a prioridefined ROIs and to a restricted number of subjects. Beside these drawbacks, there is a lack of sensitivity to detect and assess diffuse spatially distributed alterations in regional brain structure.

The present paper demonstrates a novel, alternative approach for detecting structural differences in populations. Our method is a fully automatic intensity-based algorithm and can detect regional changes in the whole brain, not only in particular regions. This allows for large samples, with no user bias or a priori-defined ROIs. The new technique is based on the analysis of deformation fields. Deformations are obtained by a nonlinear registration routine transforming a reference brain (template) onto another brain (object). The deformation field is defined by a displacement vector in each voxel. These displacement vectors constitute the transformations required for the anatomical correspondence between the template and an object brain. After estimation of this field for each subject, we characterize differences between groups by comparing the associated deformation fields. We present a general multivariate statistical approach to analyze these deformations.

We apply our method to a large sample of schizophrenic patients and healthy volunteers to address the following questions: (i) Are low frequency deformations sensitive enough to detect regional deviations between 
groups? (ii) Can these regional patterns confirm a disturbance in the nodes of the prefrontal-thalamiccerebellar circuitry in schizophrenic patients? This model of "cognitive dysmetria" as proposed by Andreasen et al. (1998) attempts to use a single theory of disruption in a nodal network to explain the diversity of schizophrenic symptoms. These nodes are located in prefrontal regions, thethalamicnudei, and the cerebellum.

\section{METHODS}

Subjects. We studied 85 DSM-IV diagnosed schizophrenic patients (33 females, 52 males) on stable neuroleptic medication with a mean age of 36.2 years (SD \pm 10.9 ). The patients exhibited primarily negative symptoms (SANS: $44.95 \pm 21.5$, SAPS: $20.88 \pm 17.3$ ). The control group consisted of 75 healthy volunteers (22 females, 53 males) with a mean age of 31.2 years (SD \pm 9.0$)$ and a negative history of major psychiatric disorders. All patients and controls were right-handed, free of any neurological disorders, and all gave written informed consent.

Scanning procedure. Sagittal T1-weighted MRI scans were obtained on a 1.5 T Philips Gyroscan ACSI I system (Matrix 2563, isotropic voxel size $1 \mathrm{~mm}^{3}$, FOV $256 \mathrm{~mm}, \mathrm{~T}_{\mathrm{R}} / \mathrm{T}_{\mathrm{E}} / \alpha=13 \mathrm{~ms} / 5 \mathrm{~ms} / 25^{\circ}$ ).

Normalization. Images were normalized using the SPM 99d spatial normalization routines (J . Ashburner and K. Friston, submitted). First, images were smoothed with an isotropic Gaussian kernel (full width at half maximum-F WHM of $8 \mathrm{~mm}$ ) and an affine transformation with 12 parameters was applied to all images to normalize them to Talairach space (Talairach and Tournoux, 1988). The transformed images were resized to a voxel size of $2 \mathrm{~mm}$ and scalp-edited by masking with a probability image of cerebral tissue. In this way, extracerebral parts of the brain were excluded to eliminate the confounding effects from scalp differences. The following nonlinear registration used is based on a regularized minimization process of residual squared difference between an image and a template of the same modality, assumed to be in Talairach space. A linear combination of $11 \times 13 \times 10$ three-dimensional discrete cosine transform basis functions was estimated to perform this registration (J . Ashburner and $\mathrm{K}$. Friston, submitted).

As a result, we obtained a deformation field for each subject with a specific three-dimensional displacement vector in each voxel. This vector describes the transformations required to map a voxel of the template brain onto its corresponding position in the object brain.

General Linear Model. For statistical analysis of the deformation fields we consider the multivariate general linear model in each voxel (Seber, 1984)

$$
\underset{(n \times d)}{Y}=\underset{(n \times p)(p \times d)}{X} \quad \underset{(n \times d)}{X}
$$

where $\mathrm{Y}$ is the matrix of $\mathrm{n}$ independent observations on $d$ response variables, $X$ contains the $n \times p$ explanatory variables (so-called design matrix), and $B$ is the matrix of $p \times d$ unknown parameters. The errors $U$ are independent and identically distributed (i.i.d.) ddimensional normal random variables with mean 0 and dispersion matrix $\Sigma$, i.e. $U \stackrel{\text { i.i.d. }}{\sim} \mathrm{N}_{d}(0, \Sigma)$.

A vast range of experimental designs can be imple-

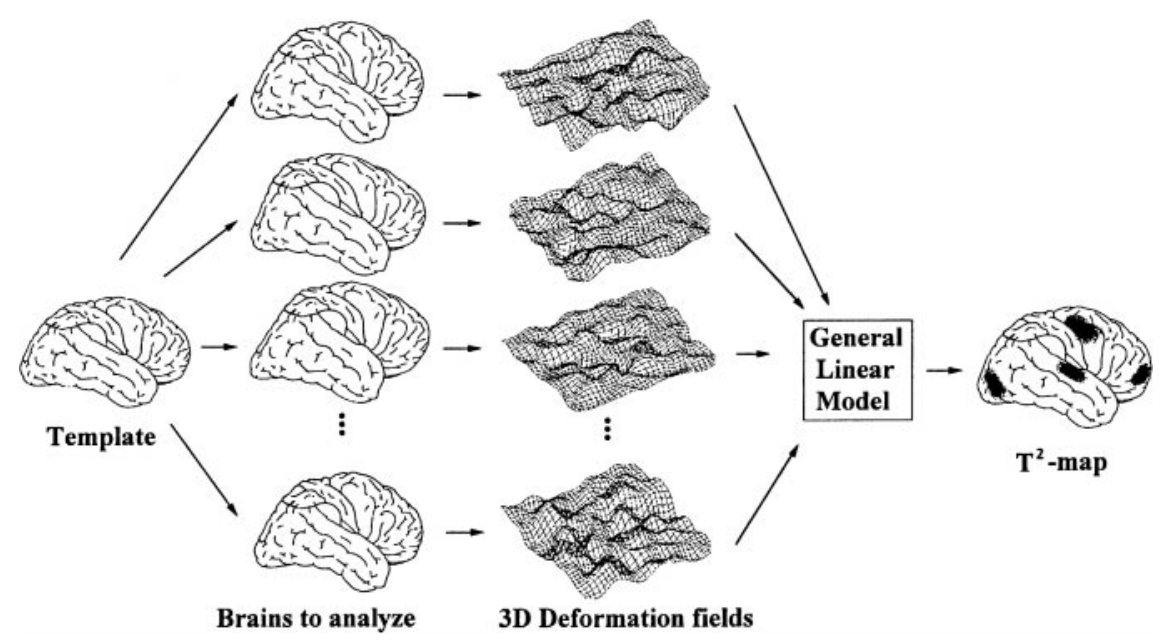

FIG. 1. This scheme describes the process of detecting structural brain changes. A template brain is spatially transformed onto each object brain using intensity-based nonlinear registration routines. As a result of this registration, we derive a deformation field for each brain which defines a 3D displacement vector in each voxel. Statistical analysis of these deformations through a multivariate general linear model results in a $\mathrm{T}^{2}$-map characterizing regional differences in brain structure. 
mented by proper specification of the design matrix $X$. If the $X$ columns contain indicator variables we can specify subtractive designs (modeling group differences) or factorial designs (interaction between factors). In a parametric design the $\mathrm{X}$ matrix consists of covariates that might explain theobserved variance in $Y$.

In addition, we can divide the design matrix into columns modeling effects of interest and noninterest (confounds). To distinguish between these effects we partitioned the matrices $\mathrm{X}=\left(\mathrm{X}_{1}, \mathrm{X}_{2}\right)$ and $\mathrm{B}=\left(\mathrm{B}_{1}, \mathrm{~B}_{2}\right)$. Thus, the general linear model is expressed as

$$
\underset{(n \times d)}{Y}=\underset{\left(n \times p_{1}\right)\left(p_{1} \times d\right)}{X_{1}}+\underset{\left(n \times p_{2}\right)}{B_{1}} \underset{\left(p_{2} \times d\right)}{B_{2}}+\underset{(n \times d)}{U},
$$

where $X_{1}$ is a $n \times p_{1}$ matrix of rank $p_{1}$, and $X_{2}$ is of dimension $n \times p_{2}$ with rank $p_{2}$ consisting of confounding variables modeling the effects of no interest. Columns of $X_{1}$ and $X_{2}$ are chosen to be linearly independent.

In terms of the model described, we use a simple subtractive design with one confounding variable to compare groups of schizophrenics and controls. This special case is equivalent to the Hotelling's T 2-Test for comparing the means of two populations. Here, $\mathrm{X}_{1}$ consists of two columns, which indicate whether an element of $Y$ is from the first group, taking the value one and otherwise zero. $X_{2}$ is a constant vector modeling the effect of equal means.

The least-squares estimates of $B$ and $B_{2}$ (Eqs. 1 and 2) are given by

$$
\hat{B}=\left(X^{\prime} X\right)^{-1} X^{\prime} Y \text { and } \hat{B}_{2}=\left(X_{2}^{\prime} X_{2}\right)^{-1} X_{2}^{\prime} Y \text {. }
$$

To obtain statistical inference on the effects of interest, we tested the null hypothesis $\mathrm{H}_{0}$ : $\mathrm{B}_{1}=0$ as follows: the error sum of squares and products (SSE) is given by the fitted values $Y$

$$
\mathrm{SSE}=(\mathrm{Y}-\mathrm{X} \hat{\mathrm{B}})^{\prime}(\mathrm{Y}-\mathrm{X} \hat{\mathrm{B}}),
$$

where the error sum of squares and products under the null hypothesis (SSH) is obtained by

$$
\mathrm{SSH}=\left(\mathrm{Y}-\mathrm{X}_{2} \hat{\mathrm{B}}_{2}\right)^{\prime}\left(\mathrm{Y}-\mathrm{X}_{2} \hat{\mathrm{B}}_{2}\right)
$$

The degrees of freedom for SSH and SSE are

$$
\mathrm{m}_{\mathrm{H}}=\mathrm{p}_{1} \text { and } \mathrm{m}_{\mathrm{E}}=\mathrm{n}-\mathrm{p}_{1}-\mathrm{p}_{2}
$$

and the likelihood ratio statistic gives Wilk's $\Lambda$

$$
\Lambda=\frac{|\mathrm{SSE}|}{|\mathrm{SSH}|} .
$$

Since $m_{H}=1$, we can transform $\Lambda$ exactly to a $\mathrm{T}^{2}$-statistic

$$
\mathrm{T}^{2}=\mathrm{m}_{\mathrm{E}} \frac{1-\Lambda}{\Lambda},
$$

which is equivalent to the common Hotelling's T2-Test for comparing the means of two populations.

Volumechange Theresulting $\mathrm{T}^{2}$-map provides information only on the significance of regional differences of deformations. We compute the J acobian determinant of the displacement vector in each voxel to specify whether change in each voxel is due to volume reduction or enlargement (Davatzikos et al., 1996). Values of the J acobian determinant $>1$ indicate volume enlargement, whereas values $<1$ point to a volume reduction. If we compute the difference of the mean J acobian determinant between schizophrenics and controls, we can obtain information on mean volume reduction and enlargement between both groups.

\section{RESULTS}

The resulting voxel-wise $T^{2}$-statistic is shown in two different formats. Figure 3 shows a maximum intensity projection, which gives an overview about all significant differences. This figure describes the maximum $\mathrm{T}^{2}$-value of all slices in three orthogonal sections. Figure 4 additionally offers the distinction between differences caused by volume reduction (colors ranging from yellow to red) or enlargement (bluecolor) in brains of schizophrenics. Both $\mathrm{T}^{2}$-maps have a threshold at a height of $\mathrm{T}^{2}=17.31\left(\mathrm{P}=0.001 ; \mathrm{T}_{3,156}^{2}\right)$ and spatial extent of $k=110$ voxels.

The most significant result was observed bilaterally in the thalamus, but more pronounced on the left side. In the frontal lobe, both hemispheres were affected. On the left side, the superior frontal gyrus and the precentral gyrus were found to be changed, while on the right side of the middle frontal gyrus was altered. In the temporal lobe, we found differences bilaterally in the superior temporal gyrus.

Furthermore, there were significant changes in the basal ganglia (right putamen), the occipital lobe (left lingual gyrus), and the left cerebellar hemisphere.

The structural differences between brains of schizophrenics and controls were mainly caused by volume reduction in the brains of schizophrenics. The only regions of volume enlargement in the brains of schizophrenics were observed in the right putamen and in the adjacent white matter of the thalamic region (Fig. 4). 

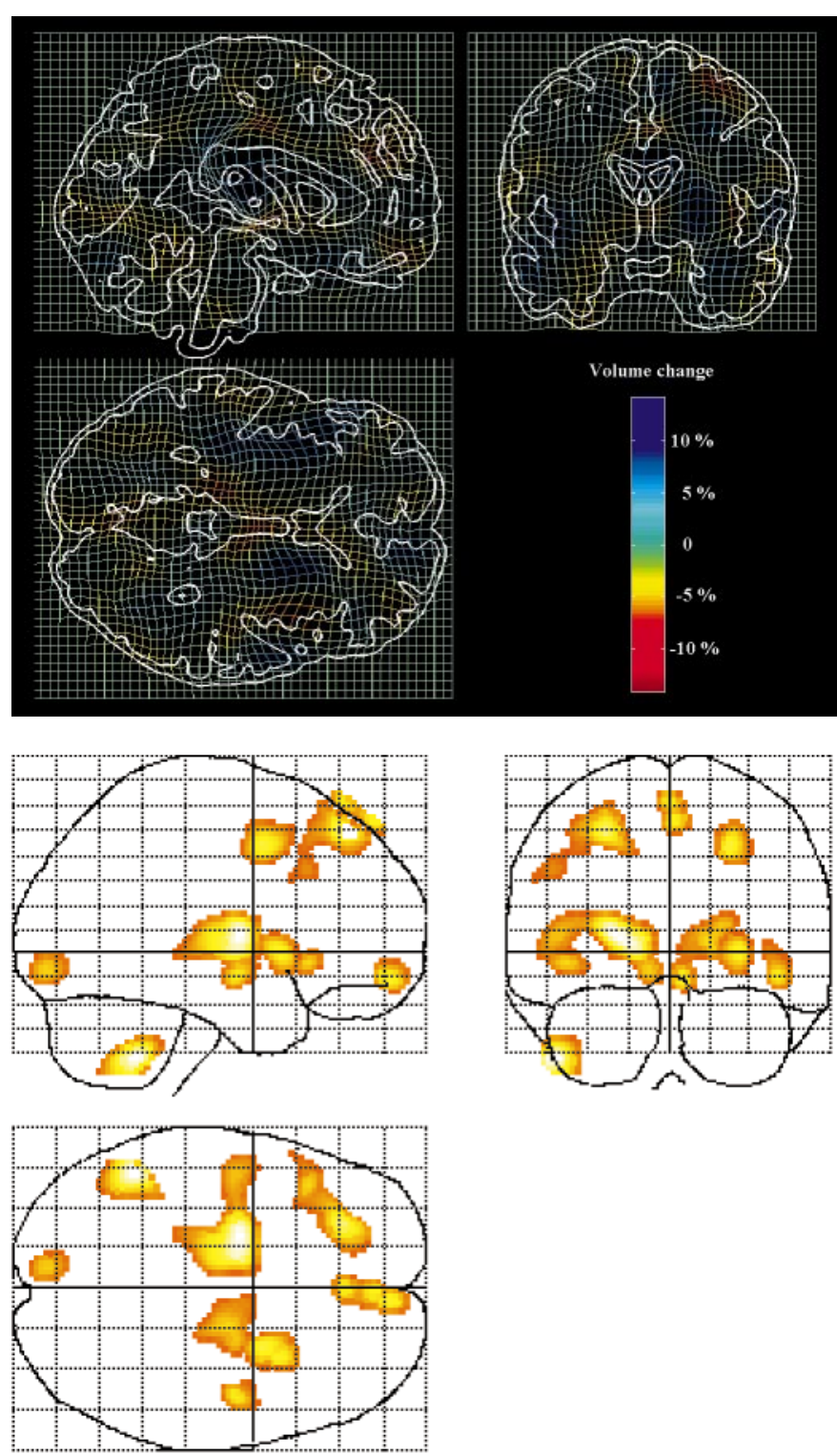

FIG. 2. This figure illustrates the mean difference in the deformations between brains of schizophrenics and controls. Section coordinates conform to Talairach atlas: $\mathrm{x}=-10 \mathrm{~mm}, \mathrm{y}=-10 \mathrm{~mm}$, and $\mathrm{z}=2 \mathrm{~mm}$. The underlying template brain is shown in three orthogonal sections as a contour plot in white. The color-coded overlay visualizes the mean difference of the deformation fields by applying the transformations to a regular grid resulting in a distorted grid (magnified by a factor of 4). A color range from yellow to red is used for regions found to be volume reduced in the brain compared to the template. Areas with vol ume enlargement are shown in colors ranging from light blue to blue.

FIG. 3. Result of the comparison between 85 schizophrenics and 75 controls. The $T^{2}$-map is displayed as maximum intensity projection in sagittal, coronal, and transverse planes. The structural differences were obtained by statistical analysis of deformations required to match the template brain onto each object brain. The map has a threshold at a height of $T^{2}=17.31(P=0.001)$ and spatial extent of $k=110$ voxels. The col orscale is arbitrary and the space conforms to that of Talairach atlas.

\section{DISCUSSION}

\section{Methodology}

The approach described here demonstrates a new method for detection of regional differences in structures in the whole brain. The technique relies on the examination of deformation fields obtained through an intensity-based nonlinear registration routine. Thestatistical analysis of these deformations by use of a multivariate general linear model results in a $\mathrm{T}^{2}$-map, which characterizes alterations in brain structure in different subjects.

Modern approaches to computational neuroanatomy that aim to identify anatomical differences fall into one 


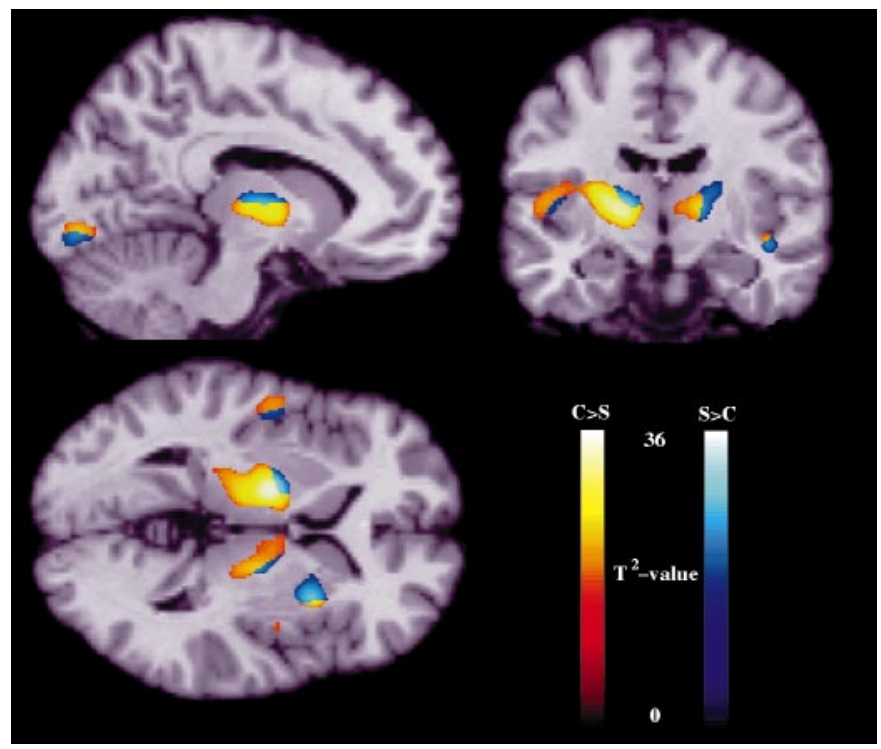

FIG. 4. The same $T^{2}$-map as presented in Fig. 3 is sectioned in three orthogonal planes and superimposed on the template image of the same anatomical space. Section coordinates conform to Talairach atlas: $x=-10 \mathrm{~mm}, y=-10 \mathrm{~mm}$, and $\mathrm{z}=2 \mathrm{~mm}$. Colors ranging from red to yellow are used for voxels found to be volume reduced in the brains of schizophrenics compared to controls. Voxels with volume enlargement are shown in blue. Significant differences are visible in the thalamus, right putamen, left superior temporal gyrus, and left lingual gyrus.

of two classes: The first addresses small volumetric changes or regionally specific changes in the amount or density of a particular tissue compartment using high resolution MRI scans. These analyses allow a voxel by voxel comparison of regional gray matter density (Wright et al., 1995), image intensity (Andreasen et al., 1994), or probability of segmented structures (Paus et al., 1996; Penhune et al., 1996).

The second complimentary class of approaches deals with the deformations of the nonlinear warping themselves and addresses anatomical changes at a larger spatial scale. The parametric form of the deformations facilitates a quantitative description of local variability in subjects along with a multivariate statistical analysis. This has been referred to as deformation-based morphometry (Ashburner et al., 1998). Deformationbased morphometry itself can be divided into those approaches that aim to characterize spatial modes of anatomic variability over the entire deformation field and those that seek to identify regionally specific differences in the deformation fields. The approach described in this paper represents the second example of the latter that employs fully 3D nonlinear deformation fiel ds. It is incidently al so among the first examples of multivariate statistical parametric mapping and extends common Hotelling's T 2-Test (Cao and Worsley, 1998; Thirion et al., 1997; Thompson et al., 1997; Worsley et al., 1996).

The applied nonlinear registration is based on the minimization of squared intensity differences between the images. It does not use landmarks or surfaces.
Smooth deformations are described by a linear combination of low spatial frequency basis functions. Hence, differences in the deformations do not directly provide information about different shapes or sizes of anatomical structures and may only partly coincide with these features. To further characterize these deformation differences, the J acobian determinant was used as a local measure whether the change is due to volume enlargement or reduction. This may be helpful to visualize in an explorative way differences in mean volume change between both groups and to distinct between areas caused mainly by growing or shrinking of local volume.

Our proposed multivariate general linear model can be applied to any deformation-based method (for an overview of nonlinear warping, see Toga and Thompson, 1999). It accommodates most forms of experimental designs such as subtractive, parametric, and factorial designs and is only restricted to the number of the covariates of interest. Because the described transformation to the $\mathrm{T}^{2}$-distribution (Eq. 8) is valid only for $m_{H}=1$ (if matrix $X_{1}$ has rank 1 ), we can use one covariate of interest. Thus, we can model the comparison of two populations or the relationship to one variable in a population. For more complex designs where $m_{H}=2$, we can transform $\Lambda$ to the F-distribution. But for higher values of $m_{H}$ there exist only approximations to $\mathrm{F}$ - or $\chi^{2}$-distributions.

It should be kept in mind that the estimation of P-values of the structural differences presented in this work is only valid when combined with an a priori 
anatomical hypothesis. In our case, we clearly specified the hypothesis of prefrontal-thalamic-cerebellar circuitry (Andreasen et al., 1998), which is a well-known hypothesis in schizophrenia research.

Generally, to use our method for an exploratory study of structural changes without an a priori anatomical hypothesis, one has to adjust the estimated $\mathrm{P}$-values to account for the multiple comparison problem. Cao and Worsley (1998) have proposed a method to estimate these adjusted $P$-values based on theE uler characteristic of the excursion set of the Hotelling's T ${ }^{2}$-test near local extrema. The application of this technique on a function of our assessed deformation fields would yield adjusted P-values for voxel height inference levels.

\section{Application to Schizophrenia Research}

Alterations of brain structures in schizophrenics have received particular attention in both neuropathological postmortem and in vivo neuroimaging studies. In spite of the inconsistencies mentioned, the prefrontal cortex has long been the focus of these investigations. However, most imaging studies have assessed only the vol ume of the whole frontal lobe or the volume of gray and white matter. They are incapable to distinguish between different subregions or even single gyri. Hence, subtle changes in subregions or diffuse spatial alterations could not be detected in these studies.

The thalamus, on the other hand, has recently gained increasing interest. Although the relatively indistinct boundaries in MRI impose difficulties on measurements, MRI studies have reported changes in this structure (Andreasen et al., 1994; Buchsbaum et al., 1996). In line with Andreasen's study, we found not only volume reduction in the thalamus itself, but also volume increase in the adjacent white matter. This suggests a compensatory process due to reduced thalamic volume.

Apart from these results, this is the first major structural MRI study to include a large sample to demonstrate systematical morphometric alterations in the cerebellum of schizophrenics. This is an important finding, particularly in the light of increasing interest in cognitive functions of the cerebellum (Leiner et al., 1995).

Taken together, the differences in every single node of the prefrontal-thalamic-cerebellar circuitry indicate a disturbance in nodes of this network. The spatial pattern of structural abnormalities in our findings provides strong additional support to the "cognitive dysmetria" theory, insofar as the central nodes of the postulated network were found to be reduced in schizophrenics (for a detailed discussion of the psychiatric impact of these findings, see Volz et al., submitted).

The conclusion of our results are threefold: (i) We have demonstrated the application of nonlinear image registration by detecting structural changes in the whole brain. This technique is fully automatic, thus allowing for the inclusion of large samples, with no user bias or a priori-defined ROIs. (ii) The framework of the multivariate general linear model covers a vast range of morphometric designs. I ts application to other deformation based techniques is straightforward and facilitates voxel based analysis of structural data. (iii) Our findings regarding the comparison between schizophrenics and controls indicate a disturbance in the nodes of the prefrontal-thalamic-cerebellar circuitry in schizophrenic patients.

\section{REFERENCES}

Andreasen, N. C., Arndt, S., Swayze, V., Cizadlo, T., Flaum, M., O'Leary, D., Ehrhardt, J. C., and Yuh, W. T. 1994. Thalamic abnormalities in schizophrenia visualized through magnetic resonance image averaging. Science 266(5183): 294-298.

Andreasen, N. C., Paradiso, S., and O'Leary, D. S. 1998. "Cognitive dysmetria" as an integrative theory of schizophrenia: A dysfunction in cortical-subcortical-cerebellar circuitry? Schizophr. Bull. 24(2): 203-218.

Ashburner, J ., Hutton, C., Frackowiak, R., J ohnsrude, I., Price, C., and Friston, K. 1998. Identifying global anatomical differences: Deformation-based morphometry. Hum. Brain Map. 6: 348-357.

Buchsbaum, M. S., Someya, T., Teng, C. Y., Abel, L., Chin, S., Najafi, A., Haier, R. J ., Wu, J ., and Bunney, W. E. 1996. PET and MRI of thethalamus in never-medicated patients with schizophrenia. Am. J. Psychiatry 153(2): 191-199.

Cao, J ., and Worsley, K. J . 1998. The geometry of the Hotelling's T2 random field with applications to the detection of shape changes. Ann. Stat. in press.

Davatzikos, C., Vaillant, M., Resnick, S. M., Prince, J . L., Letovsky, S., and Bryan, R. N. 1996. A computerized approach for morphological analysis of the corpus callosum. J . Comput. Assist. Tomogr. 20: 88-97.

Lawrie, S. M., and Abukmeil, S. S. 1998. Brain abnormality in schizophrenia. A systematic and quantitative review of volumetric magnetic resonance imaging studies. Br. J . Psychiatry 172: 110120.

Leiner, H. C., Leiner, A. L., and Dow, R. S. 1995. The underestimated cerebellum. Hum. Brain Map. 2: 244-254.

Paus, T., Tomaiuolo, F., Otaky, N., MacDonald, D., Petrides, M., Atlas, J., Morris, R., and Evans, A. C. 1996. Human cingulate and paracingulate sulci: Pattern, variability, asymmetry, and probabilistic map. Cereb. Cortex 6(2): 207-214.

Penhune, V. B., Zatorre, R. J ., MacDonald, J . D., and Evans, A. C. 1996. Interhemispheric anatomical differences in human primary auditory cortex: Probabilistic mapping and volume measurement from magnetic resonance scans. Cereb. Cortex 6(5): 661-672.

Seber, G. A. F. 1984. MultivariateObservations. Wiley, New York.

Talairach, J ., and Tournoux, P. 1988. Co-Planar Stereotaxic Atlas of theHuman Brain. Georg Thieme Verlag, Stuttgart.

Thirion, J .-P., and Calmon, G. 1997. Deformation Analysis to Detect and Quantify Active Lesions in 3D Medical Image Sequences. Technical Report 3101, Inria.

Thirion, J .-P., Prima, S., and Subsol, G. 1997. Statistical Analysis of Dissymmetry in Volumetric Medical Images. Technical Report 3178, Inria.

Thompson, P. M., MacDonald, D., Mega, M. S., Holmes, C. J ., Evans, 
A. C., and Toga, A. W. 1997. Detection and mapping of abnormal brain structure with a probabilistic atlas of cortical surfaces. J . Comput. Assist. Tomogr. 21(4): 567-581.

Toga, A. W., and Thompson, P. M. 1999. An introduction to brain warping. In Brain Warping (A. W. Toga, Ed.), Academic Press, San Diego.

Worsley, K. J ., MacDonald, D., Cao, J ., Shafie, K. H., and Evans, A. C.
1996. Statistical analysis of cortical surfaces. In Proceedings of Second International Conference on Functional Mapping of the Human Brain, p. 108. Boston.

Wright, I. C., McGuire, P. K., Poline, J . B., Travere, J . M., Murray, R. M., Frith, C. D., Frackowiak, R. S., and Friston, K. J . 1995. A voxel-based method for thestatistical analysis of gray and whitematter density applied to schizophrenia. Neuroi mage2(4): 244-252. 